\title{
Magnetic Field Effects and Radical Pair Mechanisms in Enzymes: A Reappraisal of the Horseradish Peroxidase System
}

\author{
Alex R. Jones ${ }^{1,2}$, Nigel S. Scrutton ${ }^{2 \star}$ and Jonathan R. Woodward ${ }^{1 \star}$ \\ ${ }^{1}$ University of Leicester, Department of Chemistry, University Road, Leicester LE1 7RH U.K. \\ ${ }^{2}$ University of Manchester, Manchester Interdisciplinary Biocentre and Faculty of Life Sciences, Jackson's Mill, \\ Sackville Street, Manchester M60 1QD U.K.
}

\section{Experimental Apparatus}

To undertake experiments on the effect of magnetic fields on enzyme catalysed reactions, we have constructed a dedicated Magnetic Field Effect Stopped Flow Spectrophotometer (MFESFS). This apparatus is built around a commercially available Applied Photophysics SX.18MV-R Stopped-Flow Spectrophotometer with a number of modifications.

The mixing cell for the reaction is housed in an assembly that is constructed from stainless steel and bolted onto a unit which both thermostatically controls the cell, and also injects the reagents into the cell at 8 bar pressure. A replica of the entire assembly was engineered in the workshops of the University of Leicester (UoL) Department of Chemistry and manufactured entirely from Delrin, a hard machineable plastic. This was to ensure the unit does not interact magnetically with the applied field. A pair of Helmholtz copper wound coils was produced, that mounts rigidly around the cell block and locates the observation part of the cell at the region of maximum magnetic field and greatest field homogeneity. A custom-built pulsed-current power supply was also constructed in house, which is capable of supplying a steady current pulse to the Helmholtz coils of $15 \mathrm{~A}$ for up to 5 seconds. The specific coil/current combination allows magnetic fields of up to $37 \mathrm{mT}$ to be generated, which are static over the pulse duration. The magnitude of the magnetic field pulse is varied using a personal computer via a parallel port controlled, digital to analogue converter (DAC) circuit, which allows 8-bit control over the field range. The maximum value of the field can also be scaled such that the 8-bits can span a userdefinable field range. This has the advantage of allowing detailed studies to be undertaken at low field values, which are of greater environmental consequence.

Modification was made to the Applied Photophysics control software which allows a trigger pulse to be delivered to the fieldgenerating circuit at a user-definable time $(\geq 100 \mathrm{~ms})$ prior to the firing of the stopped flow mixing unit. This is employed such that when the mixing begins, the field established at the coils is static and remains so until the kinetic measurement is complete. Thus, experiments are simply and rapidly achieved in the presence or absence of a static magnetic field of 0 to $37 \mathrm{mT}$. The field is monitored during stopped flow experiments both via measurement of the current supplied to the coils and by means of a Hall Probe (FW Bell model 5080) mounted at the radial centre on the outside edge of the outer Helmholtz coil. The field is calibrated by mounting the Hall probe at the centre of the coils (the precise cell location) with the cell removed from the assembly.

The experiments described by Grissom, Leshina and coworkers [1] were initially employed as a means of thoroughly testing this apparatus. However, the inability to reproduce the experimental results led to alternative test systems being employed. There are two key control experiments that were performed:

1) Null experiment. An experiment to ensure that the presence of the magnetic field has no effect on the measurement kinetics of a reaction in which no field effects should be observed.

2) RPM experiment. An experiment to ensure that the effect of the magnetic field can be readily measured in a reaction which is known to be magnetically sensitive through the radical pair mechanism (RPM).

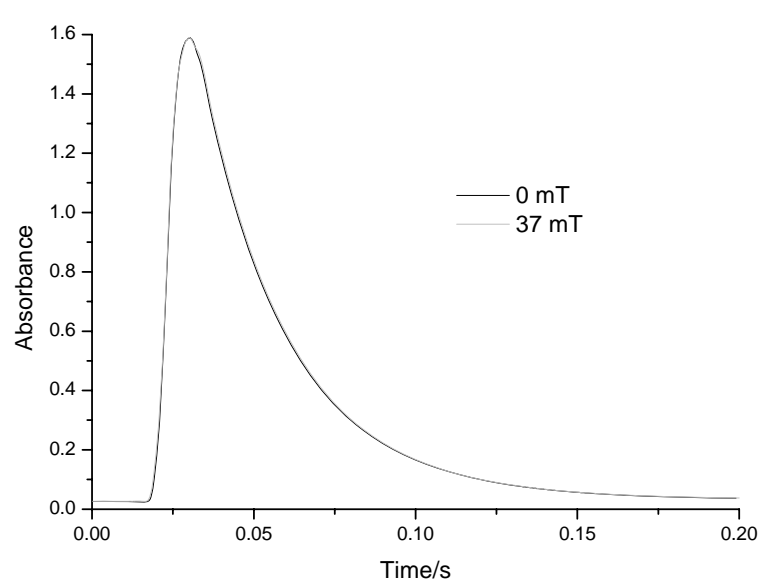

Figure 1. Transient kinetics obtained for the $524 \mathrm{~nm}$ observation of the two electron reduction of dichloroindophenol by L-ascorbic acid. No magnetic field effect is predicted in this system and none is observed. $37 \mathrm{mT}$ corresponds to the maximum field that the pulsed field apparatus can produce.

\section{Null experiment}

To ensure that the magnetic field has no instrumental effect on the measurement apparatus and does not influence the observed kinetics of a field insensitive reaction, the standard reaction used for measuring stopped flow reactor dead times was employed. This reaction is the two electron oxidation of dichloroindophenol by Lascorbic acid [2]. Figure 1 shows averaged kinetic traces for stopped flow measurements performed at $37 \mathrm{mT}$ and $0 \mathrm{mT}$ [3] for this reaction.

There is no observable difference in the kinetic traces, indicating that the magnetic field is not producing any instrumental artefacts when operated under measurement conditions

This experiment confirms that true reaction kinetics can be obtained in the presence and absence of a magnetic field in this apparatus. 
Although the field at the cell location can readily be directly measured using a Hall Effect probe, we felt it necessary to demonstrate that the apparatus be used to show a positive magnetic field effect in a test reaction system. The reaction selected was the photoexcitation of pyrene (Py) in the presence of 1,3-dicyanobenzene (DCB). This system has been used extensively in the study of RPM magnetic field effects in both laser based [3] and field modulation [3] experiments, and proceeds via the formation of a singlet radical pair $(\mathrm{RP}),{ }^{1}\left[\mathrm{Py}^{*+} \mathrm{DCB}^{\circ}\right]$. This RP further produces a singlet exciplex whose fluorescence can be monitored in the blue region of the spectrum. Singlet-triplet state mixing in the RP produces a triplet RP $\left({ }^{3}\left[\mathrm{Py}^{*+} \mathrm{DCB}^{*}\right]\right)$ which has non-radiative fates. Thus the fluorescence signal measures the amount of reaction from the singlet radical pair and the system is a useful one for observing the classic RPM field effect as the applied magnetic field retards spin-state mixing due to the energetic removal of the $T_{ \pm 1} R P$ sublevels through the Zeeman interaction.

A solution of $10^{-4} \mathrm{M}$ Py and $10^{-2} \mathrm{M}$ DCB in a 9:1 cyclohexanol : acetonitrile mixture was loaded into the stopped flow syringes of the apparatus. The MFESFS was configured such that the input fibre optic light guide (carrying UV light from the Xe arc lamp for sample excitation) and the output fibre optic light guide (carrying the fluorescence light signal to the PMT) were perpendicular. The experimental sequence then proceeded as follows:

1) The magnetic field was switched on

2) The stopped flow syringes were fired, mixing occurred in the reaction cell and the monitoring of the PMT signal commenced.

3) The magnetic field pulse ended.

4) After an appropriate delay, the monitoring of the PMT signal concluded.

The results of this experimental sequence for a magnetic field of $25 \mathrm{mT}$ are indicated in figure $2 \mathrm{~b}$. There is a very clear difference in the magnitude of the fluorescence signal reaching the PMT in the presence and absence of a magnetic field. Two further precautions were taken to ensure that this effect was a true magnetic field effect and not instrumental in origin.

1) The experiment was repeated at increasing field values from 0 to $25 \mathrm{mT}$

2) The experiment was repeated under identical conditions but with no DCB added to the reaction solution.

Figure 2a shows the MARY (MAgnetic effect on Reaction Yield) curve obtained as a result of the first experiment. There is a clear saturation of the field effect, as the RPM predicts for this reaction system. The observed $\mathrm{B}_{1 / 2}$ value is in good agreement with literature values. Thus the observed effect of the magnetic field can unequivocally be assigned to the effect on S-T state mixing through the Zeeman Effect.
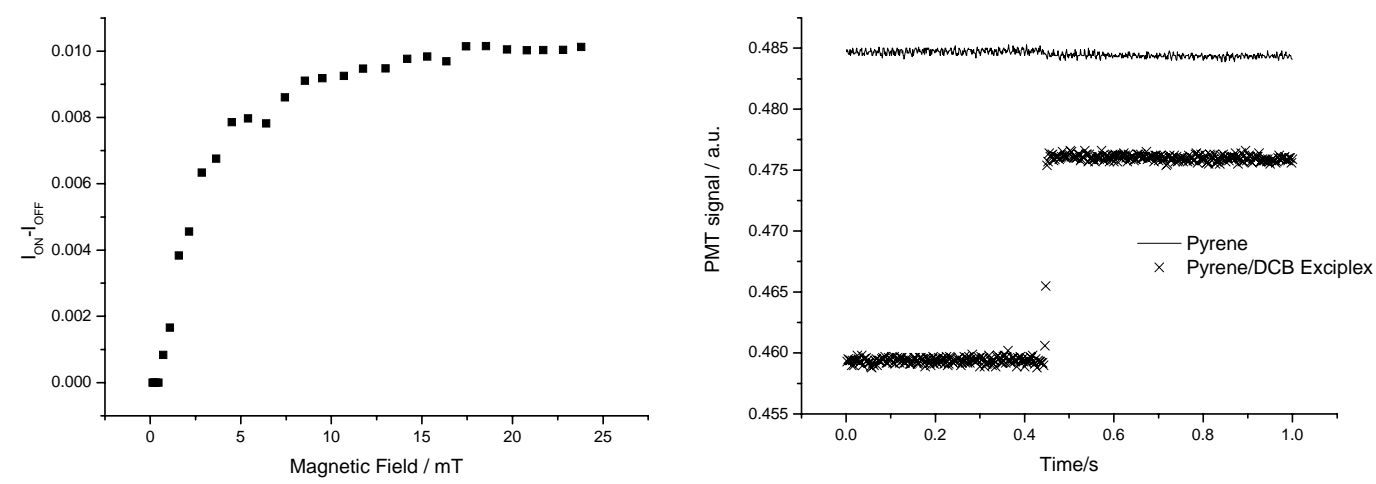

Figure 2. a) MARY curve obtained from the change in fluorescence intensity of the pyrene/DCB exciplex on application of a range of magnetic field strengths. b) Affect of a $25 \mathrm{mT}$ MF switching off during data acquisition on a pyrene $(-)$ and pyrene/DCB $(\times)$ system. Both solutions were prepared in acetonitrile:cyclohexanol, 1:9 mixture.

The results of the second experiment are shown in figure $2 \mathrm{~b}$. There is a very tiny disturbance of the fluorescence signal observed in the presence and absence of the magnetic field, but this was due to a small change in the DC response of the PMT with the magnetic field present and has subsequently been removed by the application of a mu metal shield.

Thus these null and RPM test experiments prove conclusively that the apparatus itself is not adversely affected by the presence of a magnetic field to any significant extent and that a magnetic field effect on a model chemical system could be readily measured, even without the use of field modulation.

\section{Summary of the previous findings of Grissom, Leshina and co-workers [1].}

The previous MFE study of horseradish peroxidise monitored the decay kinetics of the HRP catalysed reduction of hydrogen peroxide, using the enolate of 2-methylpropanal (generated from 2-methyl-1-(trimethylsilyloxy)-1-propene) as a reductant, at $418 \mathrm{~nm}$. Scheme 1 in the main article text shows the reaction cycle. Native HRP is rapidly converted to Compound I within the mixing time of the instrument. Subsequently Compound I is converted to compound II in a pseudo-first order process and Compound II is converted back to native HRP in a subsequent pseudo-first order process. A kinetic fitting function was determined for this scheme (see section 4) and 
fitted to the observed experimental absorbance traces. This allowed the extraction of the net rate constants $k_{1}$ and $k_{2}$, which correspond to the conversion of Compound I to Compound II and Compound II to native HRP respectively.

The original experiments determined zero field values of $k_{1}$ and $k_{2}$ as $6 \mathrm{~s}^{-1}$ and $20 \mathrm{~s}^{-1}$ respectively. The reaction was performed in a series of different strength magnetic fields and the changes in $k_{1}$ and $k_{2}$, reported as relative rate coefficients, are given in Table 1.

Table 1. Relative values for $k_{1}$ and $k_{2}$ at a range of magnetic field strengths, as reported by Grissom, Leshina and co-workers [1].

\begin{tabular}{|c|c|c|}
\hline Magnetic Flux (mT) & $k_{1}$ relative rate coefficient $\left(\mathrm{s}^{-1}\right)$ & $k_{2}$ relative rate coefficient $\left(\mathrm{s}^{-1}\right)$ \\
\hline 0 & 1 & 1 \\
\hline 1 & $1.033 \pm 0.020$ & $1.288 \pm 0.069$ \\
\hline 10 & $0.976 \pm 0.048$ & $1.056 \pm 0.123$ \\
\hline 25 & $0.959 \pm 0.021$ & $0.960 \pm 0.059$ \\
\hline 50 & $0.860 \pm 0.020$ & $0.713 \pm 0.054$ \\
\hline 75 & $0.841 \pm 0.027$ & $0.656 \pm 0.069$ \\
\hline 100 & $0.955 \pm 0.019$ & $0.855 \pm 0.054$ \\
\hline 200 & $1.029 \pm 0.028$ & $1.110 \pm 0.098$ \\
\hline 300 & $1.045 \pm 0.025$ & $1.107 \pm 0.081$ \\
\hline
\end{tabular}

In the previous study, anticipated magnetic field effects were calculated using the semiclassical approximation of magnetic field dependent radical pair recombination and were in good agreement with the observed effects on $k_{1}$ and $k_{2}$.

\section{Reproduction of the HRP experiments}

The experiments described in [1] were performed in our newly constructed MFESFS adhering to the original conditions whenever possible. There are some typographical errors in the original manuscript, but in consultation with the authors of the original work, the reaction conditions were confirmed as:

- All solution concentrations quoted are after mixing within the reaction cell. All reagent solutions were prepared in $100 \mathrm{mM}$ $\mathrm{KH}_{2} \mathrm{PO}_{4}$ buffer, titrated to $\mathrm{pH} 7.4$ with $\mathrm{KOH}_{(\mathrm{aq})}$.

- Horseradish Peroxidase (Sigma Type IV), $1.4 \mu \mathrm{M}$, determined spectrophotometrically using $\varepsilon=102 \mathrm{mM}^{-1} \mathrm{~cm}^{-1}$ at $403 \mathrm{~nm}$.

- Hydrogen Peroxide, $140 \mu \mathrm{M}$, prepared fresh daily and determined spectrophotometrically using $\varepsilon=39.4 \mathrm{M}^{-1} \mathrm{~cm}^{-1}$ at $240 \mathrm{~nm}$.

- 2-Methyl-1-(trimethylsilyloxy)-1-propene (Aldrich), $16 \mathrm{mM}$, was solubilised in ethanol (concentration $2.43 \mathrm{M}$ ) before mixing with buffer. The phosphate mediates the rapid hydrolysis of the substrate to the free enolate [4] (the active form). Under these reaction conditions, the enolate is present at a steady state concentration of about $1.3 \times 10^{-7} \mathrm{M}$.

- The substrate and hydrogen peroxide solutions were loaded together in the left-hand drive syringe, with the HRP solution in the right. All solutions were passed through a $0.45 \mu \mathrm{m}$ filter immediately prior to use.

- $\quad$ Temperature $=25^{\circ} \mathrm{C}$.

- $20 \mu \mathrm{l}$ quartz reaction cell of $10 \mathrm{~mm}$ optical path length.

The experimental apparatus in our experiments differed from the original work in three ways that are significant, but should have no effect on the observed kinetics.

1) In the original work, the stopped flow mixing cell was placed between the poles of a permanent electromagnet which delivered magnetic fields of up to $300 \mathrm{mT}$. In our experiment, the maximum field achievable is $37 \mathrm{mT}$. Also, in our experiment, the magnetic field is pulsed, but is constant throughout the monitoring period of the reaction.

2) The original work employed a rapid scanning spectrophotometer which allowed a range of analysis wavelengths to be monitored in a single experiment. All data analysis, however, only employed the data obtained at $418 \mathrm{~nm}$. All kinetic data in the original work contains high frequency (non-random) noise on the absorbance signal generated due to the rapid scanning nature of the device. Our experiment is a true single wavelength experiment (with monochromation pre-sample) that produces data with a higher signal-to-noise ratio.

3) The path length of the cell in our apparatus is $1.0 \mathrm{~cm}$ compared with $1.7 \mathrm{~cm}$ in the original experiment. Thus the changes in absorbance observed here are correspondingly smaller than the values in the original work.

\section{Experimental results.}

Our experiments were performed in the following manner.

The stopped flow syringes were loaded with the relevant reagents and were then allowed to equilibrate to the water bath temperature of $25^{\circ} \mathrm{C}$, along with the cell assembly. For each syringe fill, a maximum of 18 injections could be performed. Injections were performed alternately in the presence and absence of a magnetic field pulse, with the initial injection randomly selected as field on or field off. In all experiments a minimum of the first two field on and field off injections were discarded as they did not provide a true kinetic response. Thus, a typical experiment involved 7 field on and field off decay curve acquisitions. 


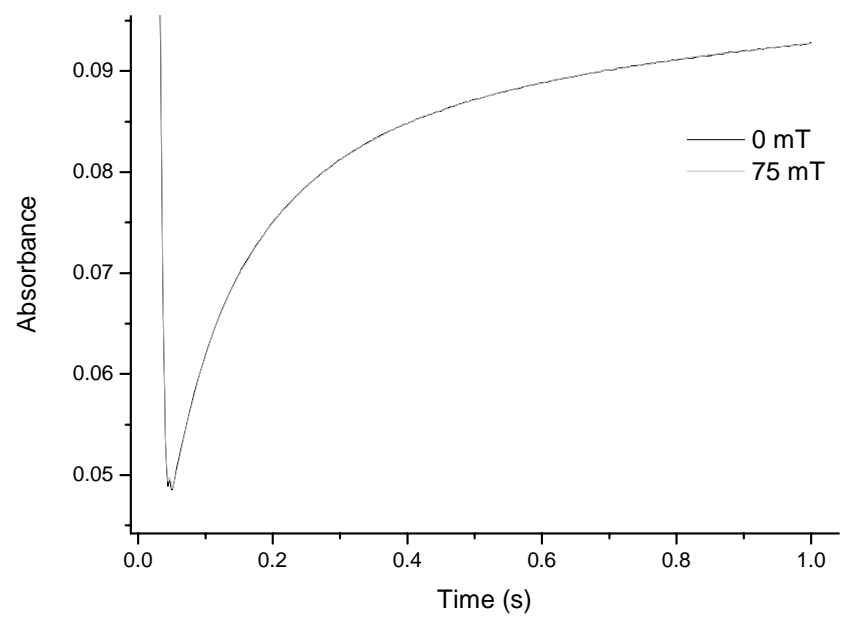

Figure 3. Absorbance traces recorded at $418 \mathrm{~nm}$ for the HRP reaction with a magnetic field on value of $75 \mathrm{mT}$. There is no observable difference in the two curves. This data represents one experiment of seven recorded for this field value.
Magnetic field values were chosen to be as close to those in the original work but were limited by the maximum attainable magnetic field of the apparatus. Thus, experiments were performed at $1,10,25$ and $37 \mathrm{mT}$. The first three are points which correspond to those used previously, and fall within the range of our apparatus. The fourth is our maximum attainable value. Multiple experiments were performed for each field value; for example 7 individual experiments were performed at 37 $\mathrm{mT}$.

The most significant MFE observed in Grissom and Leshina's work was at a field of $75 \mathrm{mT}$. As our apparatus is incapable of producing a field of this magnitude, a pair of permanent rare-earth magnets were mounted either side of the mixing cell to generate a homogeneous field of $75 \mathrm{mT}$ at the sample position. This arrangement has the benefit of allowing reinvestigation of the most critical data point and also provides an arrangement in which there is no possibility whatsoever of the field generating apparatus affecting the measurement apparatus.

The results of the experiments are represented in two ways. First, figure 3 shows the kinetic traces obtained for a single experiment at $75 \mathrm{mT}$ and is representative of the results obtained at all field values. Here the field on traces for each injection have been averaged to provide a single decay curve. The same has been done for the field off injection traces and the two kinetic traces are plotted on the same axes.

It is clear from figure 3 that the signal-to-noise ratio of the data is extremely good. It is also clear, however, that there is no observable difference in the reaction kinetics at $0 \mathrm{mT}$ and $75 \mathrm{mT}$. This result is confirmed by a more detailed statistical analysis. The absorbance data was fit using a kinetic model (see section 4) and decay parameters $k_{1}$ and $k_{2}$ were extracted from the data. The relative rate coefficients were elucidated for each field on/off pair, and the mean values and standard deviations for each experiment calculated. The overall mean of each experimental mean, and each experimental standard deviation were then calculated and are displayed in Table 2 .

Table 2. Relative values for $k_{1}$ and $k_{2}$ at a range of magnetic field strengths from this study.

\begin{tabular}{|c|c|c|}
\hline Magnetic Flux (mT) & $k_{1}$ Relative Rate Coefficient $\left(\mathrm{s}^{-1}\right)$ & $k_{2}$ Relative Rate Coefficient $\left(\mathrm{s}^{-1}\right)$ \\
\hline 0 & 1 & 1 \\
\hline 1 & $0.998 \pm 0.031$ & $0.995 \pm 0.049$ \\
\hline 10 & $0.996 \pm 0.037$ & $0.996 \pm 0.065$ \\
\hline 25 & $0.998 \pm 0.026$ & $1.005 \pm 0.036$ \\
\hline 37 & $0.998 \pm 0.049$ & $1.001 \pm 0.064$ \\
\hline 75 & $1.001 \pm 0.061$ & $0.998 \pm 0.098$ \\
\hline
\end{tabular}

There is no difference in the values of $k_{1}$ and $k_{2}$ obtained at any value of the magnetic field.

\section{Methods of analysis}

Our inability to reproduce the magnetic field effects observed in [1] led us to take a more detailed look at the means by which the analysis in the original work was performed. This is discussed in the supporting information that accompanies the publication. The absorbance data is analysed by means of a simple kinetic scheme given in scheme 1 .

Scheme 1. Reaction scheme for the observed kinetics. Compound I is produced by rapid reaction of Native HRP with hydrogen peroxide. It then reacts with enolate substrate (the concentration of which is kept constant by decomposition of the silyl enol ether precursor) in two pseudo first order steps to generate Compound II and then regenerate Native HRP.

$$
\text { Compound I } \stackrel{k_{1}}{\longrightarrow} \text { Compound II } \stackrel{k_{2}}{\longrightarrow} \text { NativeHRP }
$$

This yields the following expressions for the concentrations of Compound I, Compound II and Native HRP.

$$
[\text { Compound }]=[\text { Compound }]_{0} \mathrm{e}^{-k_{1} \mathrm{t}}
$$




$$
\begin{gathered}
{[\text { CompoundII }]=\frac{[\text { CompoundI }]_{0} k_{1}}{k_{2}-k_{1}}\left(\mathrm{e}^{-k_{1} \mathrm{t}}-\mathrm{e}^{-k_{2} \mathrm{t}}\right)} \\
{[\text { NativeHRP }]=[\text { Compound }]_{0}\left(1-\frac{k_{2}}{k_{2}-k_{1}} \mathrm{e}^{-k_{1} \mathrm{t}}+\frac{k_{1}}{k_{2}-k_{1}} \mathrm{e}^{-k_{2} \mathrm{t}}\right)}
\end{gathered}
$$

The analysis at $418 \mathrm{~nm}$ is based on the relative absorbance of these three species at this wavelength. The paper erroneously states that the extinction coefficient of Native HRP is twice that of Compound II, but according to [5], the converse is true. This appears to be a typographical error, as the equation given is correct and is obtained by adding equations 2 and 3 with a scaling of 1 and 0.5 respectively.

$$
[\text { Compound II + NativeHRP }] \approx \frac{[\text { CompoundI }]_{0}}{2}\left(1-\frac{k_{2}-2 k_{1}}{k_{2}-k_{1}} \mathrm{e}^{-k_{1} \mathrm{t}}-\frac{k_{1}}{k_{2}-k_{1}} \mathrm{e}^{-k_{2} \mathrm{t}}\right)
$$

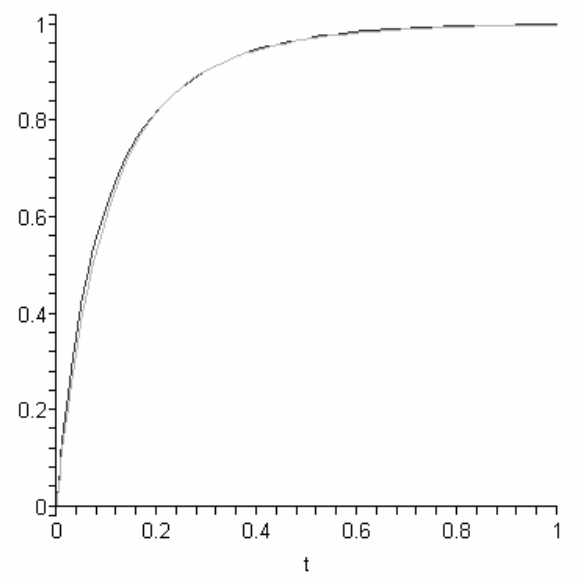

This function was then fitted to the individual absorbance curves obtained for each injection. No data was given on the quality of each fit.

In order to gauge the original observed difference in the shape of the absorbance traces in the absence and presence of a magnetic field, we worked backwards from the values of $k_{1}$ and $k_{2}$ originally reported to reproduce the curves obtained from least-squares fitting. Figure 4 shows a plot of the reconstructed fitting curves for the magnetic field that showed the greatest difference in the values of $k_{1}$ and $k_{2}$ in the presence and absence of a field, i.e. $75 \mathrm{mT}$. Under these conditions, the relative (to zero field) values of $k_{1}$ and $k_{2}$ were 0.841 and 0.656 respectively.

Figure 4. Reproductions of the kinetic fits obtained in [1] obtained by regenerating the observed absolute values of $k_{1}$ and $k_{2}$ and substituting in equation 4. It is clear that although the differences in $k_{1}$ and $k_{2}$ in the presence and absence of a field are large, there is virtually

The reconstructed functions are virtually indistinguishable from one another. There is a straightforward reason for this which is simply illustrated by observing the individual effects of $k_{1}$ and $k_{2}$ on the kinetics. This is illustrated in figure 5 .
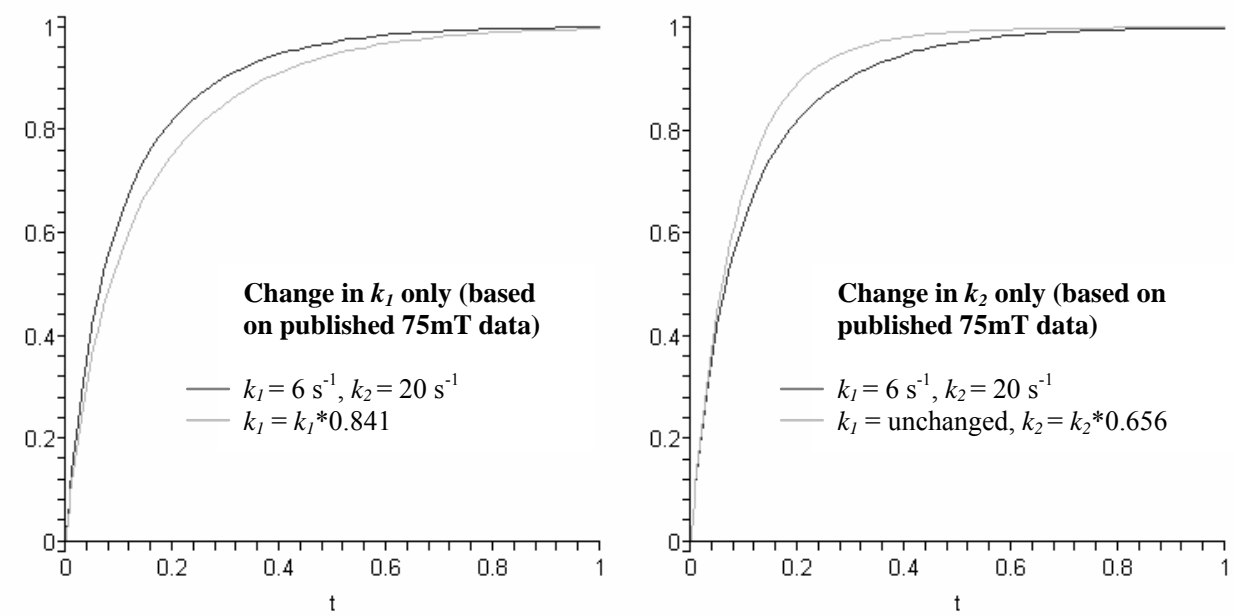

Figure 5. Effects on the shape of the kinetic fit for individual changes to $k_{1}$ and $k_{2}$. It is clear that these two parameters cause approximately opposite effects on the shape of the fit. Thus, changes in both parameters simultaneously tend to counteract one another giving little change to the overall fit.

It is clear that decreasing $k_{1}$ causes the absorbance signal to rise more slowly but that decreasing $k_{2}$ has the opposite effect. The reason for this is that the extinction coefficient of native HRP is half that of Compound II and so reducing the rate of the step that converts Compound II to Native HRP means that Compound II survives longer and the absorbance is greater. Thus the overall effect is that certain values of $k_{1}$ and $k_{2}$ can be found where the effects counteract one another. All the data points in the original work correspond to changes in $k_{1}$ and $k_{2}$ that almost precisely counterbalance one another. This may help to explain one of the problems that the authors of the original work remarked upon (reference note $\left.(15)^{1}\right)$ in that the proposed magnetic field sensitive step should lead to changes in $k_{2}$ but cannot explain effects on $k_{1}$. 
The other obvious problem with this kinetic analysis is that Compound I quite clearly has a non-negligible absorbance at $418 \mathrm{~nm}$. In fact, the extinction coefficient is approximately one third of that of Native HRP and one sixth of that of Compound II. In order to fit our own data, equation 4 was modified to include the contribution from Compound I. The precise fitting function employed is given in equation 5. The final term has been adjusted simply to scale the curve so that it can be fit to absorbance values initially scaled to zero (i.e the initial concentration of Compound I is subtracted).

$$
\begin{aligned}
& {[\text { Compound II + NativeHRP }} \\
& + \text { Compound I }]
\end{aligned} \approx \frac{[\text { CompoundI }]_{0}}{2}\left(1-\frac{k_{2}-2 k_{1}}{k_{2}-k_{1}} \mathrm{e}^{-k_{1} \mathrm{t}}-\frac{k_{1}}{k_{2}-k_{1}} \mathrm{e}^{-k_{2} \mathrm{t}}+\frac{\mathrm{e}^{-k_{1} \mathrm{t}}-1}{3}\right)
$$

Figure 6a shows a typical absorbance trace obtained from our spectrometer fit with equation 4. Figure $6 \mathrm{~b}$ shows the much improved kinetic fit obtained with equation 5 . All the data that appear in table 2 were obtained by fitting with equation 5 .
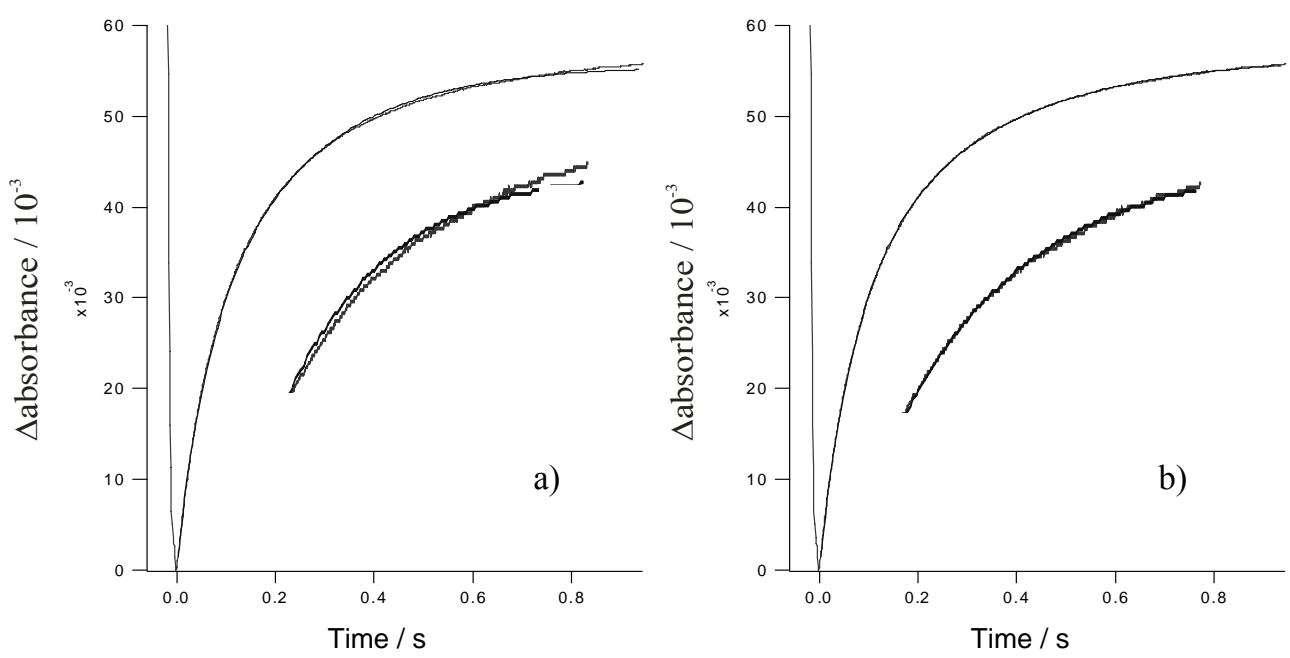

Figure 6. Quality of fit to observed absorbance data. Figure a) shows the fit obtained using equation 4 i.e. the same as that in [1]. b) shows the improved fit by the inclusion of a term corresponding to compound I (equation 5). In both case, the final part of the curve has been zoomed and inset for clarity.

It is also worth noting that this improved fitting equation shows a different dependence on the values of $k_{1}$ and $k_{2}$ and that when the values in the original work for $75 \mathrm{mT}$ are substituted into equation 5 , the field on and off decays are now distinguishable - i.e. the relative changes to $k_{1}$ and $k_{2}$ that balance the effects are different for this equation. This is as expected as only $k_{1}$ appears in the term arising from Compound I.

\section{Other issues}

It should also be pointed out that there is a possibility that the process represented in Scheme 1 of the main communication is not the only chemistry which can occur in this system. A range of work was carried out in the late seventies and early eighties by Cilento and coworkers [6] on the HRP catalysed aerobic oxidation of isobutanal, the products of which were formic acid and triplet excited acetone. It was discovered that the active species was the enolate tautomer, the same active species as in this current study. Although the comparative rate of this reaction is very slow (over the course of minutes), simply extracting $k_{1}$ and $k_{2}$ from the kinetic trace resultant from an aerobic stopped flow experiment may not be entirely accurate. It was noted in the current work, that although the majority of the absorbance change occurred within one second, it took around $100 \mathrm{~s}$ for the absorbance to rise to a stable value.

\section{References}

[1] Taraban, M. B.; Leshina, T. V.; Anderson, M. A. and Grissom, C. B., J. Am. Chem. Soc. 1997, 119, 5768-5769

[2] The field at the sample cell with no current passing through the Helmholtz coils has been measured by Hall Probe to be less than $0.1 \mathrm{mT}$

[3] Batchelor, S. N.; Kay, C. W. M.; McLauchlan, K. A. and Shkrob, I. A., J. Phys. Chem. 1993, 97, 13250-13258.

[4] Novice, M. H.; Seikaly, H. R.; Seiz, A. D. and Tidwell, T. T., J. Am. Chem. Soc., 1980, 102, 5835-5838.

[5] Yokota, K. and Yamazaki, I., Biochemistry, 1977, 16, 1913.

[6] (a) Durán, N.; Oliveira, O. M. M. F.; Haun, M.; Cilento, G. J.C.S. Chem. Comm. 1977, 66, 442 (b) Bechara, E. J. H.; Oliveira, O. M. M. F.; Durán, N.; De Baptista, R. C.; Cilento, G. Photochem. Photobiol. 1978, 39, 101 (c) Baader, W. J.; Bohme, C.; Cilento, G.; Dunford, H. B. J. Biol. Chem. 1985, 260, 18, 10217 